\title{
AN INCIDENTAL CASE OF UTERUS DIDELPHYS AND FIBROIDS: RIGHT SIDED MYOMECTOMY AND LEFT HEMI-HYSTERECTOMY
}

Mashala J.N., Wekesa D., Chemwey R., Pulei A., Kihara A.

Correspondence to Dr. Jonathan Mashala Nyime, Department of Obstetrics and Gynecology, University of Nairobi. P.O. Box: 19676-00202 Nairobi Kenya. Tel +254748826513. Email: jonamashala@yahoo.co.uk

\begin{abstract}
Congenital anomalies of the uterus occur due to agenesis or lack of fusion of the Mullerian ducts. The incidence of these anomalies is largely unknown since they are unlikely to be diagnosed in asymptomatic women. Uterine didelphys or double uterus occurs when the two Mullerian ducts fail to fuse resulting in duplication of the uterus and cervix. This report discusses a case of a 38-yearold woman who had presented with a longstanding history of abnormal uterine bleeding and cyclical pain. She had had two successful vaginal deliveries prior to presentation to our unit. Sonographic findings revealed large uterine myomas and was consented for open myomectomy. Intraoperatively she was found to have two uteri, both of which had intramural fibroids and two cervices.
\end{abstract}

Keywords: didelphys uterus, fibroid.

\section{INTRODUCTION}

Mullerian duct anomalies are congenital defects of the female genital system that arise from abnormal embryological development of the Mullerian ducts. These abnormalities can include failure of development (Heinomen, 1984; Golan et al., 1989), fusion with absence of canalization or reabsorption, which normally occurs between 6 and 22 weeks in utero. When classifying these anomalies solely based on abnormal development, four major types are apparent: Complete or partial failure of Mullerian duct development (agenesis; unicornuate uterus without a rudimentary horn) (Golan et al., 1989; Grimbizis et al., 2001); Failure of ducts to canalize (unicornuate uterus with a rudimentary horn without proper cavities; Incomplete fusion of
Mullerian ducts (bicornuate or didelphys uterus); Incomplete reabsorption of uterine septum (septate or Arcuate uterus) (Jena et al., 2015 ). Didelphys uterus has been estimated to occur in $1 / 3000$ women (Lopamudra et al., 2015). Most women are asymptomatic, but some present with dyspareunia or dysmenorrhea in the presence of a varying degree of longitudinal vaginal septum. Rarely, genital neoplasms, hematocolpos/hematometrocolpos, and renal anomalies are reported in association with didelphys uterus (Wunderlich, 2007). However, in some cases of women with a didelphys uterus they do not exhibit any reproductive or gestational challenges.

\section{CASE REPORT}

A 38 year old Para 2+0 Kenyan of African descent was admitted to Kenyatta National Hospital (KNH) through the gynecology outpatient clinic (GOPC) with complaints of abdominal pain and discomfort for 2 weeks. In 2012, she had consulted at the $\mathrm{KNH}$ in the GOPC where she was diagnosed with symptomatic uterine fibroid with associated menorrhagia. In November 2017, upon clinical examination, she was found to have a big, irregular, firm, and non-tender mass arising from the pelvic region. A pelvic sonogram showed a solitary intramural fibroid in posterior myometrium measuring $8.38 \mathrm{~cm}$ by $8.06 \mathrm{~cm}$, and a pedunculated fibroid on the left adnexa measuring $13.84 \mathrm{~cm}$ by $11.93 \mathrm{~cm}$. As she desired fertility, she was scheduled for myomectomy. She was admitted and signed informed voluntary consent for the procedure. The surgery was performed within the same month. Intra-operatively, an incidental finding of didelphys uterus was made. There was no prior history of pregnancy-related or reproductive complications before the year 
Anatomy Journal of Africa. 2020. Vol 9 (2): 1762 -1764.

2012. Myomectomy was successfully conducted on the right uterine horn.

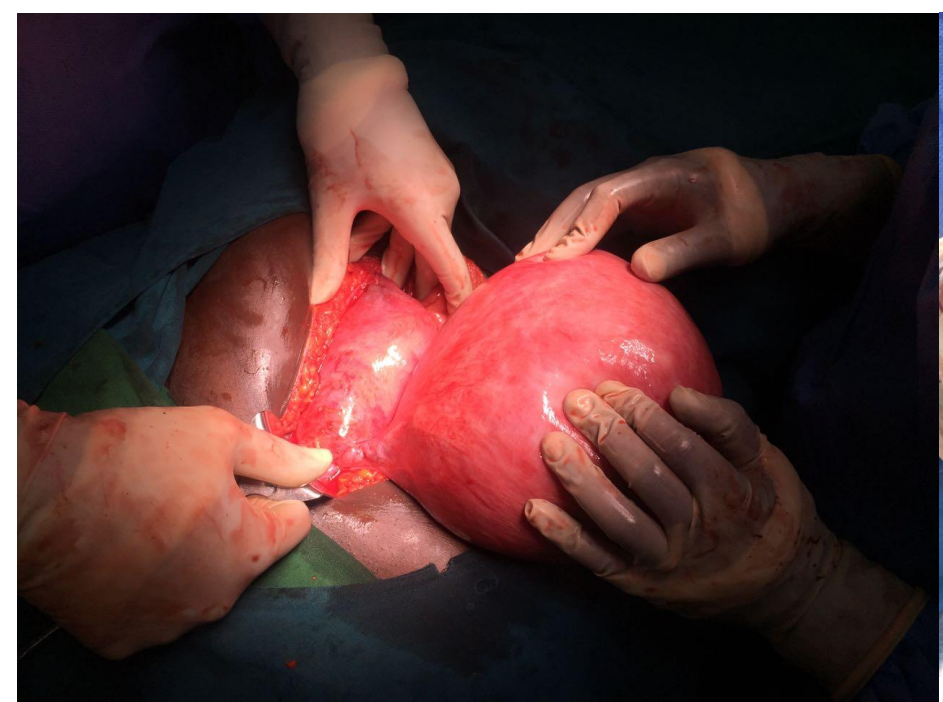

Figure 1: Didelphys uterus with left sided huge fibroid visualized

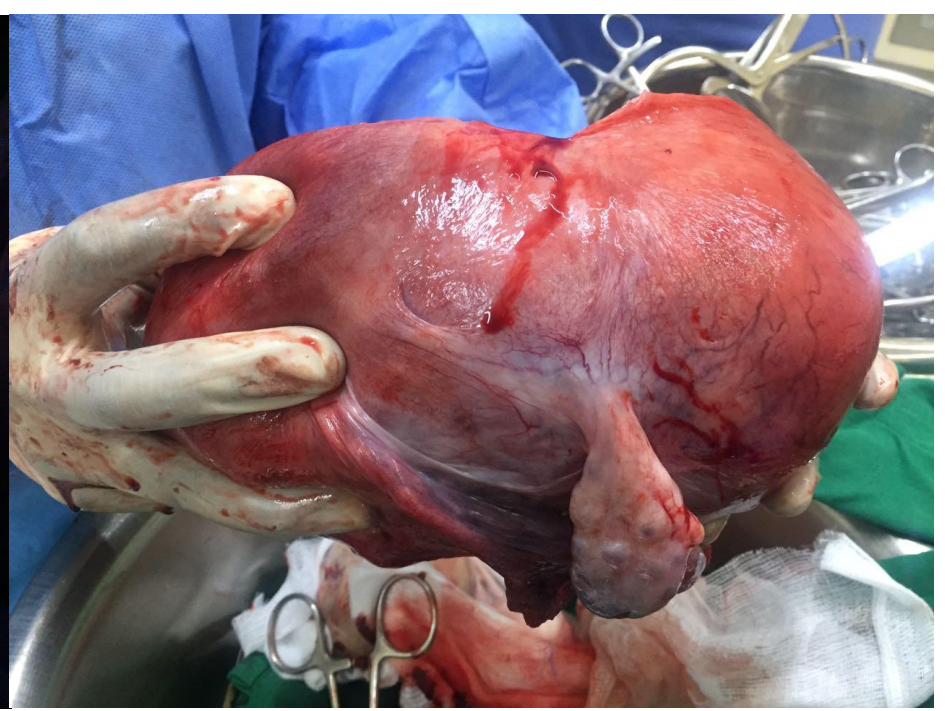

Figure 2: The left uterus with huge fibroid and left adnexa visualized.

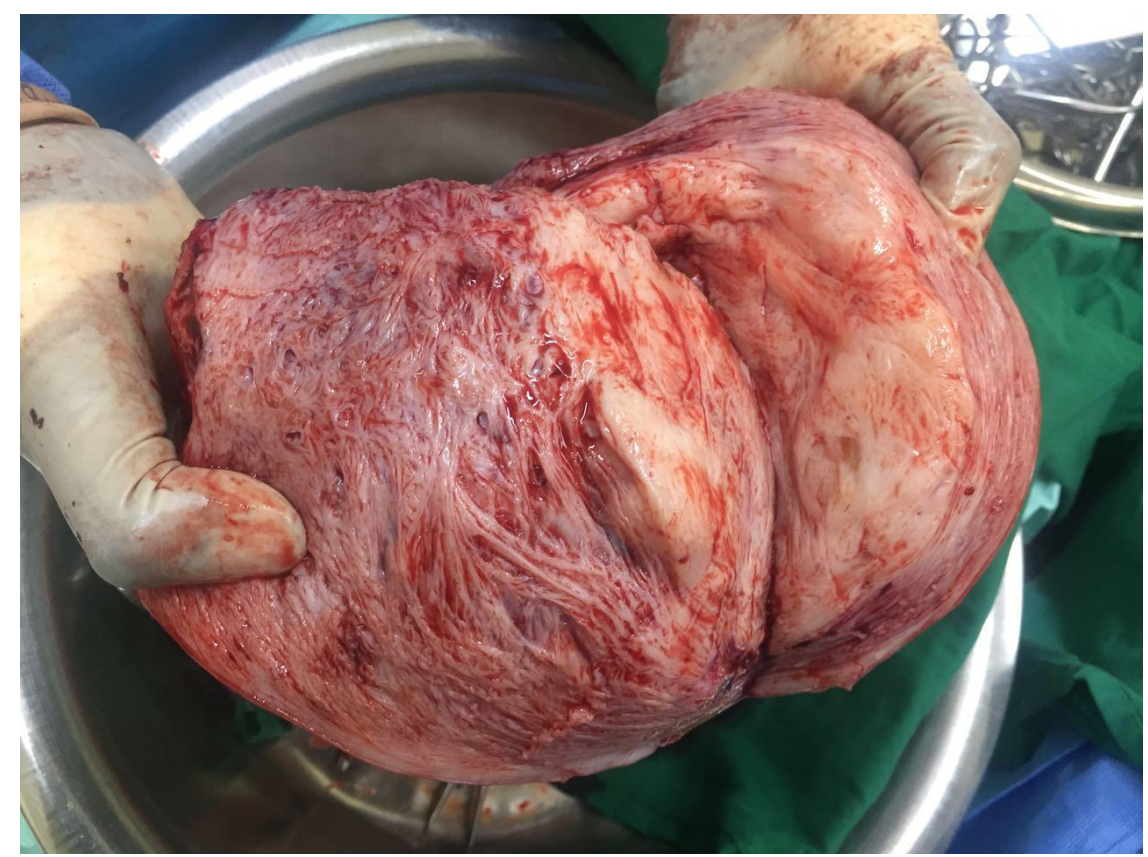

Figure 3: The left sided hemi-hysterectomy specimen incised revealing the huge fibroid.

\section{DISCUSION}

A didelphys uterus is a rare condition that develops in female fetuses. Normally, the two Mullerian ducts are fused together to create a single uterus, but occasionally these ducts develop into two separate uteri with double cervices and potentially double vaginas (Khairy et al., 2013). They uteri are both usually functional with reported cases of women giving birth to twins, each housed in the separate uterine horns (Khairy et al.,
2013). There is a reported case of a virgin patient with a didelphys uterus with multiple fibroids and consequent infertility where total hysterectomy with bilateral removal of adnexa was performed. There are very few similar cases reported in the literature. Generally, reported cases present with infertility and presence of fibroid masses was thought to contribute to this patient's problem (Khairy et al., 2013; Strassmann, 1961; Strassmann, 
1966; Speroff and Mitchell, 2005). However, in this case the patient was $\mathrm{P} 2+0$ and had carried her pregnancies to term without any complications and deliveries were uneventful. In this patient right-sided myomectomy was performed and left sided hemi hysterectomy and adenectomy was performed.

In conclusion, a didelphys uterus is a very rare Mullerian duct anomaly with varying reproductive and gestational outcomes. The ability to conceive remains challenging. There is insufficient data on surgical corrective methods such as (metroplasty) and therefore it is not usually indicated (Strassmann 1961; Strassmann 1966). Unique to this case, a combination of myomectomy on the right horn with conservation of the right adnexa was done, and a left hemi-hysterectomy and left adenectomy performed. There is paucity of evidence indicating management of a similar case additionally. It is also advisable to look for an obstructed Mullerian system whenever a multicystic dysplastic kidney or the absence of a kidney is discovered in a fetus or girl post-natally. A high index of suspicion with pelvic pathologies co-existent with Mullerian and renal anomalies necessitate cautious approach to affirming the diagnosis and the surgical interventions undertaken to avert long-term morbidity or mortality (Jacques et al., 1992).

\section{REFERENCES}

1. Heinomen PK. 1984. Uterus didelphys; a report of 26 cases, European journal of obstetrics \& gynecology and reproductive biology. 17:345-350.

2. Golan A, Langer R, Bukovsky I. 1989. Congenital anomalies of the Mullerian system. Fertile sterile. 51(5): 747: 55.

3. Grimbizis GF, Camus M, Trarlatzis BC, Bontis, BN, Devroey P 2001. Clinical implications of uterine malformations and hysteroscopic treatment results hum reprd update. 7: 161- 174.

4. Jena L, Puhan JN, Swain S, Kanungo S. 2015. Department of obstetrics \& gynecology, SCB Medical college, Cuttack, Odisha, 2015. India uterine didelphys: a rare case report. Thesis.

5. Lopamudra Jena, Jyoti Narayan Puhan, Sujata Swain, Shyama Kanugo Uterine didelphys: a rare case report 2015 India.

6. Wunderlich HW 2007. Syndrome uterus didelphys, blind hemi vagina and ipsilateral renal agenesis sonographic and MR findings in 11 cases, 37:657- 665.

7. Khairy Ali MK, Abdelbadee AY, Shazly SAEM, Abbas MS 2013. A case report on Uterus didelphys with multiple fibroids.

8. Strassmann EO. 1961. Fertility and unification of double uterus and endometrial atresia clin abstet gynecol, 4: 240

9. Strassmann EO. 1966. Fertility and unification of double uterus. Fertile Sterile, 17(2): 16576

10. Speroff L, Mitchell C. 2005. Clinical Gynecologic Endocrinology and infertility $.7^{\text {th }}$ ed. Philadelphia, Pa: Lippincott Williams and Wilkins. Pg. 113.

11. Jacques P, Stassart MD, Theodore C, Nagel MD, Konald A. 1992. Fertility and sterility, uterus didelphys, obstructed hemi vagina, and ipsilateral renal agenesis, the University of Minnesota experience, 57(4): 756- 761. 